\title{
Role of infection in the death of children with acute lymphoblastic leukaemia
}

\author{
A. W. CRAFT, M. M. REID, E. BRUCE, J. KERNAHAN, AND P. S. GARDNER \\ From the Departments of Child Health, Haematology, and Virology, Royal Victoria Infirmary, and \\ University of Newcastle upon Tyne
}

SUMmaRY Twenty-four consecutive deaths from a total of 70 children receiving treatment for acute lymphoblastic leukaemia (ALL) have been reviewed. An attempt has been made to ascribe the cause of death to either infection, haemorrhage, the leukaemia itself, or a combination of these factors. No child was free of infection at death. Infection, with or without haemorrhage, was responsible for the deaths of all 15 children whose leukaemia had not relapsed. Although infection was present at death in all 9 children whose leukaemia had relapsed, the leukaemia process itself was also a major contributing factor. Viruses were associated with death in many of the children and may be emerging as important pathogens in children with ALL. Familiarity with a protocol may be an important factor in the prevention of fatal infections in such children. Centralization of treatment is necessary if this expertise is to be acquired.

The use of combination drug therapy and the prophylactic treatment of the central nervous system (CNS) in children with acute lymphoblastic leukaemia (ALL) has resulted in many patients surviving for more than 5 years (Holland and Glidewell, 1972; Simone et al., 1975). However, prolonged survival has been accompanied by the risk of severe infection in these children who, at times during the course of their treatment, may have impaired immune responses. In order to determine the part played by infection in the death of children with ALL, the last 24 consecutive deaths in such patients treated with combination chemotherapy and CNS prophylaxis in Newcastle upon Tyne have been reviewed. An attempt has been made to ascribe the cause of death to either infection, haemorrhage, the leukaemia itself, or a combination of these factors. Any rational improvement in treatment must be based on an accurate knowledge and explanation of past failures, so in reviewing these deaths we hope to be better able to plan future management.

Patients, management, and methods

Most of the children with ALL in the Northern Regional Health Authority Area are now referred to one of the two major hospitals in Newcastle upon Tyne for treatment. From a total of 70 children in whom a diagnosis of ALL was made during the

Received 15 February 1977 period December 1972 to November 1976 there were 24 deaths, occurring between March 1973 and August 1976. 15 of these deaths occurred either during the induction phase of treatment (4) or during the first remission (11) (group I); the remaining 9 children (group II) died after a first or subsequent haematological relapse and all had blast cells in the peripheral blood during their terminal illness. Details of these two groups are given in Tables 1 and 2. The patients are ranked according to the time interval between diagnosis and death.

Three of the 24 patients were treated with one of the Medical Research Council's UKALL regimens (Medical Research Council Working Party, personal communication, 1976; MacLennan et al., 1976), while the remaining 21 were treated with a slightly modified version of the best arm of the Acute Leukaemia Group B (ALGB) 6801 protocol (Holland and Glidewell, 1972). The latter consisted of induction with vincristine and prednisone, weekly intrathecal methotrexate during the 5-week induction phase followed by cranial irradiation with 2400 rads in 12 doses over 19 days, maintenance with continuous 6-mercaptopurine and weekly methotrexate, and intermittent 'pseudo-reinductions' with vincristine, prednisone, and intrathecal methotrexate. The first 7 patients in group I did not survive long enough to receive cranial irradiation.

General supportive care consisted of red cell and platelet transfusions and appropriate antibacterial or antifungal therapy when indicated. Case 7 
Table 1 Patients dying during induction phase or in first remission of ALL (group I)

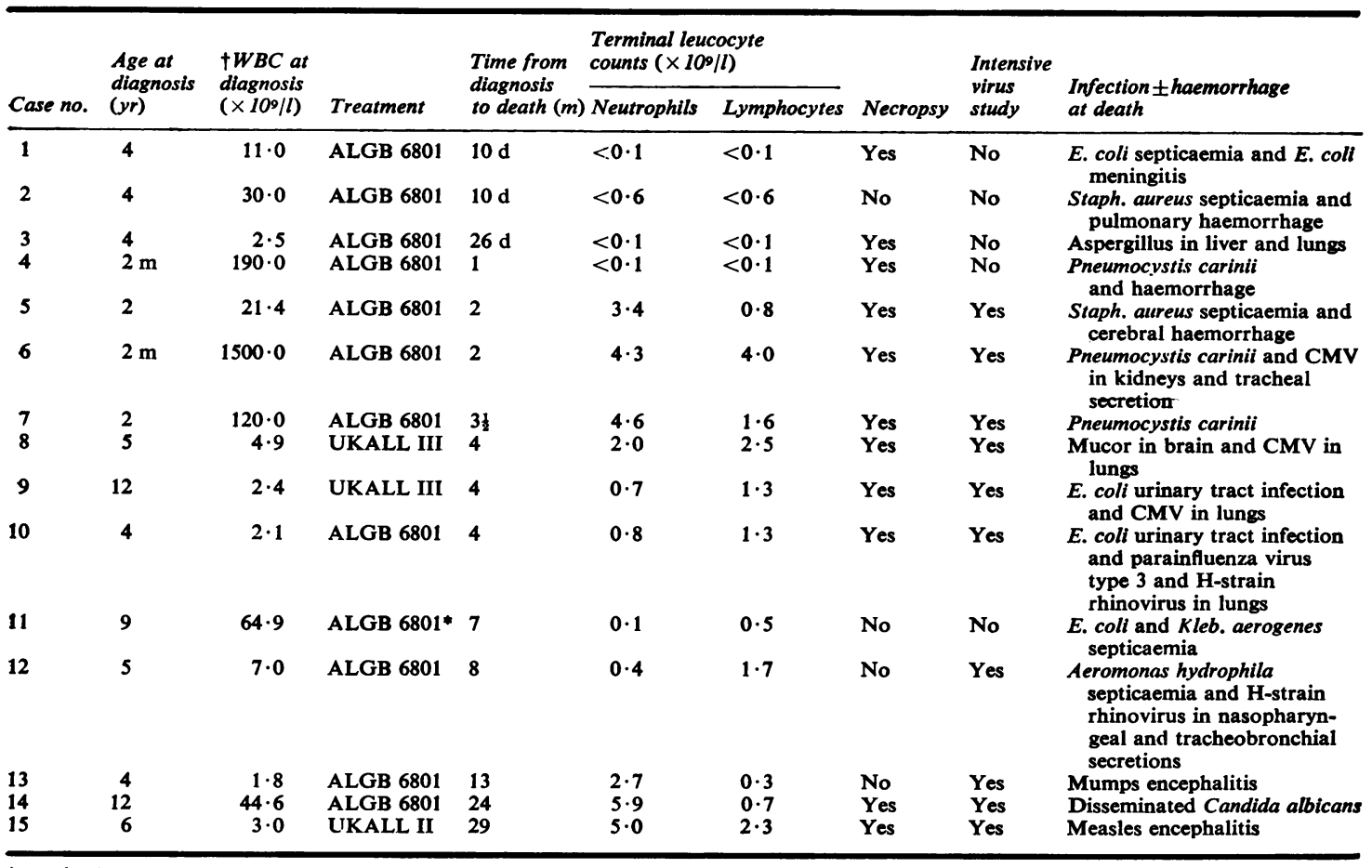

-Received L-asparaginase and cytosine arabinoside, in addition, to induce remission.

+White blood cell count

Table 2 Patients dying after relapse of $A L L$ (group II)

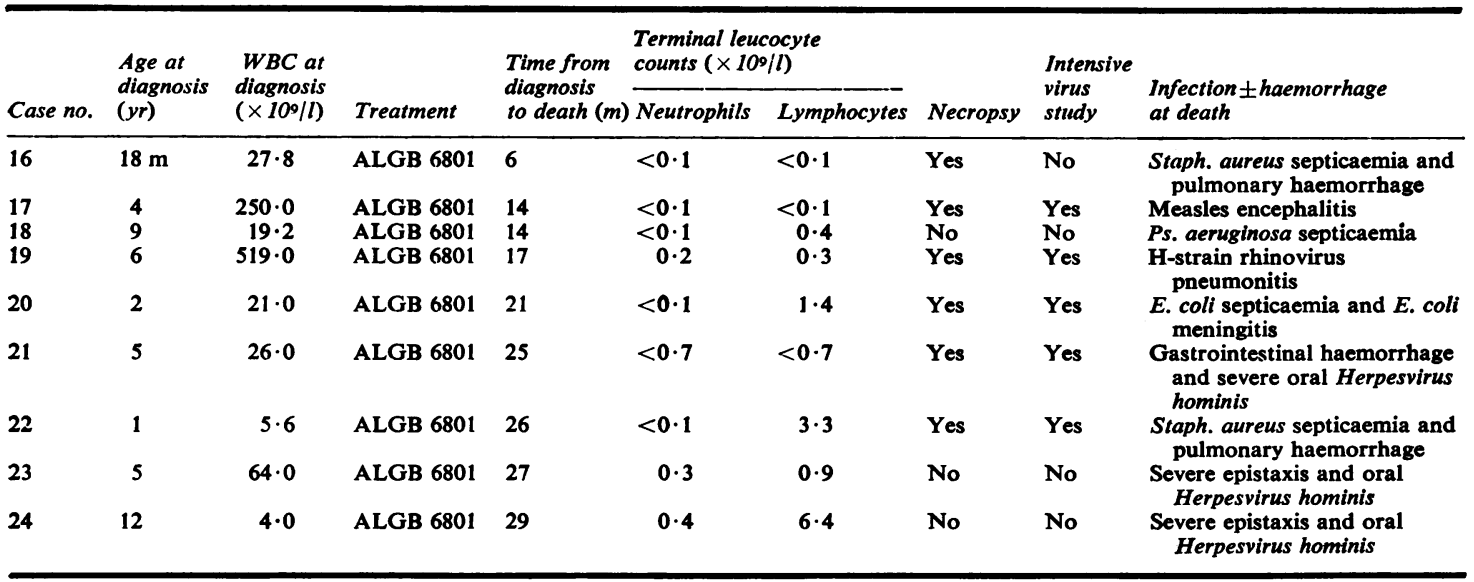

received pentamidine for Pneumocystis carinii pneumonitis diagnosed by a percutaneous needle lung biopsy. White cell transfusions were not available in Newcastle until February 1976, but 2 patients (Cases 1 and 3) were transferred to Glasgow for this form of therapy and have been included in the review by Graham-Pole et al. (1976). Since this facility has been acquired two episodes of infection have been successfully treated. Strict reverse-barrier nursing or other isolation procedures were not routinely employed during the early phase of this study, but since July 1975 isolation of children either by barrier 
nursing or under laminar air flow has been practised during episodes of neutropenia with counts of less than $0.5 \times 10^{9}$ neutrophils/1.

During the early phase of the study there was a longer observation period before treating suspected infections with antibiotics but more recently these have been given at an earlier stage. Most ill children with a pyrexia of more than $38^{\circ} \mathrm{C}$ were assumed to have a bacterial septicaemia and received broad spectrum parenteral antibiotics until the results of blood cultures were known. Appropriate changes in antibiotic therapy were then made.

The major findings at the time of death are based on clinical observation, ante-mortem investigation, and, where possible, on evidence obtained from necropsy examination. During the early part of this study period, necropsy examination consisted of routine morbid anatomy and histology, but since October 1974 intensive virological examination, both before and after death, has also been made. This consists of examination of specimens from many organs by immunofluorescent techniques and viral culture methods which have been fully described elsewhere (Gardner et al., 1967; Gardner and McQuillan, 1974). Details of 3 of the deaths, Cases 5,15 , and 17, have been reported previously (Sims et al., 1976; Pullan et al., 1976).

\section{Results}

In group I infection was present at death in every child and in $12(80 \%)$ was the only reason for death. In the remaining 3 severe haemorrhage was present in addition to infection. In group II, all of whom had blast cells in the peripheral blood terminally, infection was again present in every child. 5 of the 9 also had severe haemorrhage. 2 of these patients, Cases 23 and 24, did not have complete ante-mortem investigation of their terminal illness in order to avoid further distress to themselves and their families. All four fatal staphylococcal infections were associated with haemorrhage, 3 with severe pulmonary haemorrhage, and 3 deaths in which Herpesvirus hominis was involved were associated with severe gastrointestinal or nasal haemorrhage.

Fig. 1 shows the terminal neutrophil and lymphocyte counts according to the 'predominant' type of infection present at death. The counts from 4 children are not included. It proved too difficult to assign cases 9 and 10 to a single infection group and cases 23 and 24 were not adequately investigated during their terminal illness. In those children with a bacterial infection there is a trend towards lower neutrophil counts than in the other infection groups. There are no significant differences between the absolute lymphocyte counts in the four groups of infections.

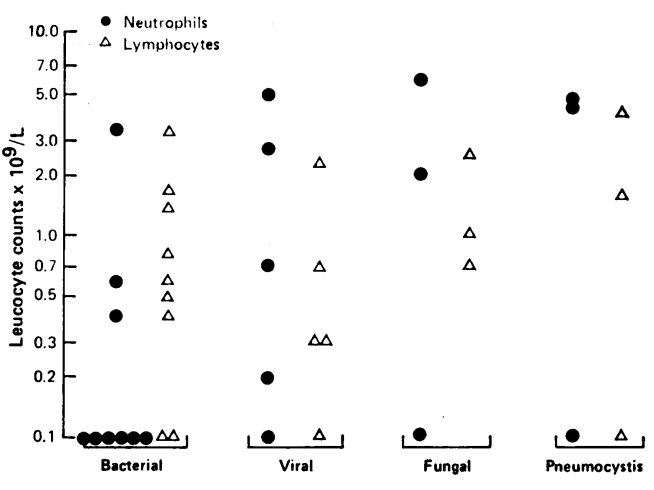

Fig. 1 Terminal leucocyte counts in the 'predominant' infection groups.

A total leucocyte count at diagnosis of more than $20 \times 10^{9} / 1$ and/or aged 12 or more years was taken as indicating a bad prognosis. Table 3 shows the distribution of cases with good and bad prognosis in the whole group of patients and in the deaths. The results suggest that in the good prognosis group death from infection in remission is a greater hazard than relapse.

Table 3 Distribution of cases with good and bad prognosis

\begin{tabular}{lcc}
\hline Patients & Good prognosis $(n)$ & Bad prognosis $(n)$ \\
\hline All patients $(\mathrm{n}=70)$ & $43(62 \%)$ & $27(38 \%)$ \\
Deaths in remission or & $7(47 \%)$ & $8(53 \%)$ \\
during induction $(\mathrm{n}=15)$ & $7(22 \%)$ & $7(78 \%)$ \\
Deaths in relapse $(\mathrm{n}=9)$ & $2(2 \%)$ \\
\hline
\end{tabular}

A necropsy examination was performed in 17 of the 24 children. All of the bacterial infections had been confirmed by ante-mortem blood cultures except in Cases 9 and 10 in group I who both had $E$. coli urinary tract infections 2 days before death. The virus infections were confirmed either by culturing the virus from post-mortem specimens or by histological appearances. Pneumocystis carinii pneumonitis was diagnosed in life in one child, but in the other 2 only by examination of lung sections. The aspergillus and mucor infections were shown histologically and the Candida albicans by culture from many sites at necropsy examination.

\section{Discussion}

This study confirms previous findings that infection is often present at death in children with ALL. Hughes (1971) found that of 199 children with leukaemia, infection was responsible for death in 89 $(45 \%)$ and only $44(22 \%)$ were completely free of 
infection. Of the 8 deaths in remission reported in the UKALL II trial (MacLennan et al., 1976), 6 $(75 \%)$ were associated with infection, while in UKALL I (CNS irradiation) (Medical Research Council, 1975) all 5 deaths in remission were associated with infection. In our series no child was free of infection at death. Infection, with or without haemorrhage, can reasonably be claimed to be the cause of death in the group I patients. In group II, however, the effect of the leukaemic process itself must be taken into account and in these patients it might be reasonable to claim that they all died of 'leukaemia'. The apparent increased frequency of infection in our series may simply reflect more intensive searching for organisms both before and after death.

In Fig. 1 the division of the children into 'predominant' infection groups does not imply certainty of the cause of death. It merely reflects a decision based on a combination of clinical judgement and microbiological and virological evidence in the light of what is already known about the particular organisms. Some children with more than one organism present at death may have been placed in an inappropriate group due to our lack of knowledge of the relative importance of each organism. Except in Case 5, all the bacterial infections were associated with neutropenia. 4 of the 5 deaths in the viral infection group were associated with lymphopenia. The fifth, Case 15, had a prolonged measles encephalitis which in its early stages was associated with a lymphocyte count of $0.6 \times 10^{9} / 1$, but which by the time of his death had reached normal levels.

Opportunistic infections with organisms usually of low pathogenicity have frequently been cited as a major cause of infection in immunosuppressed patients (Feigin and Shearer, 1975). In our series only one of the bacterial infections, that due to Aeromonas hydrophila, is usually considered to be in this category and has previously been reported as causing a fatal infection in leukaemia (Pearson et al., 1972). However, two of the three fungal infections did involve such organisms. Mucor and aspergillus are both organisms well recognized as pathogens in the compromised host. Although the virus infections cannot be classed as opportunistic, the clinical picture was different from that normally seen in the uncompromised host. Measles, mumps, rhinovirus, and cytomegalovirus (CMV) do not cause such devastating illness in normal subjects. The 2 children who had measles encephalitis have recently been reported (Pullan et al., 1976). A leading article (British Medical Journal, 1976) reported 11 cases of this encephalopathy which had been reported up to 1976 and further cases have recently been reported from the United States (Murphy and Yunis, 1976).
Pneumocystis carinii pneumonitis has become common in the South-west United States but until recently it had not been a clinical problem in this country. Many major centres are now seeing an increasing number of patients with this infection which may in part be due to better diagnosis, but it is also possible that more intensive chemotherapy is an important contributing factor. In only one of our patients was the condition diagnosed in life, but this was too late for treatment to be effective. Treatment with cotrimoxazole or pentamidine is successful in many patients in whom an early diagnosis can be made and prophylactic cotrimoxazole may be effective in preventing pneumocystis pneumonitis from occurring (Hughes, 1976).

Viruses have been found in the lungs or respiratory tract secretions of several of the children at death. Their presence is probably abnormal but their contribution to death is sometimes difficult to determine. A study of all childhood deaths now in progress in Newcastle may clarify the position. However, as many nonleukaemic childhood deaths are associated with respiratory tract illness, it may be some time before a clear picture emerges. Case 19, who died in relapse and whose lungs at necropsy examination contained $\mathrm{H}$-strain rhinovirus, had the histological appearances of interstitial pneumonicis. However, Case 10, who died in remission with a urinary tract infection and parainfluenza virus type 3 and rhinovirus in her lungs, had no typical pneumonitis changes. This difficulty in ascertaining the relative importance of virus infections histologically is due to the poor cellular response and minimal inflammatory exudate in some of these patients. H-strain rhinovirus may be emerging as an important pathogen in children with ALL. CMV pneumonitis, whose clinical appearance may resemble pneumocystis pneumonitis, has been shown to be a cause of death in immunosuppressed patients (Abdallah et al., 1976).

The interaction of different types of infection is not well understood. Case 12 died of a bacterial infection associated with rhinovirus in nasopharyngeal and tracheobronchial secretions 4 months after a prolonged and severe attack of measles with giant cell pneumonitis. Some virus infections may suppress immune responses and render the host liable to invasion with other organisms. The finding of impaired neutrophil function in normal children with virus infections supports this view (Craft et al., 1976)

Similarly, the effects of the interaction of antileukaemic drugs and radiotherapy on the immune mechanism are poorly understood. 4 of the remission deaths, those of Cases 8,9,10, and 11, occurred soon after radiotherapy and this phenomenon has been noted in the UKALL trials (MacLennan et al., 1976). 
A marked fall in the lymphocyte count with a relatively greater depression of B lymphocytes after radiotherapy (Esber et al., 1976; Reid et al., 1977) may, in part, account for this increased susceptibility to infection.

It is worth emphasizing that almost two-thirds of the deaths in our series were due to infection in patients whose leukaemia had not relapsed. The deaths during remission induction $(5.7 \%)$ are rather high but if the baby (Case 4) is excluded this is reduced to $4.3 \%$ which is comparable to the $3 \%$ in the study of Hughes and Smith (1973) but slightly higher than in the UKALL I study where it was $2 \%$ (Johnston et al., 1974). Fortunately, the number of patients who did suffer a relapse is small and the overall survival continues to be encouraging. Within any treatment regimen there needs to be a balance between obtaining the best control of the disease and incurring severe infection. Prevention of these deaths during remission and induction would significantly improve survival figures.

Two approaches are available to combat the problem of infection in these patients. One can try to both prevent infection and improve the methods of treatment of established infections. Fig. 2 shows the

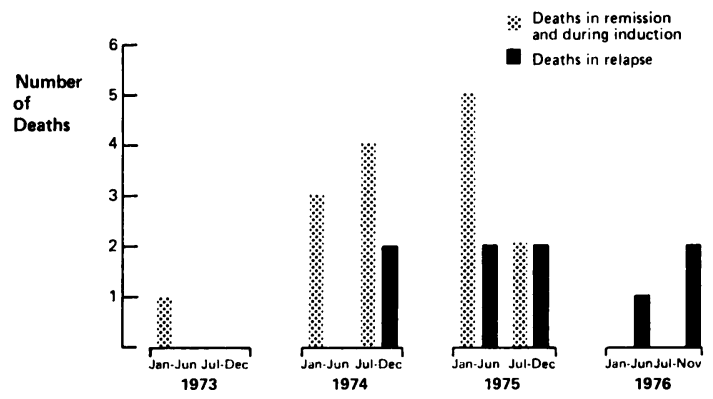

Fig. 2 6-month periods in which deaths occurred. The study period includes only 11 months of 1976. The last death in remission occurred in October 1975.

6-month periods in which the deaths in our series occurred. The decreasing number of patients in remission dying of infection in the later part of the study and the absence of any deaths in remission for over 1 year despite a steadily increasing population of children being treated for ALL suggests that familiarity with a protocol may be an important factor in the prevention of fatal infections.

Protective isolation and gut sterilization are possible in certain centres but the amount of increased nursing work that these entail may be formidable. It is difficult to assess the contribution of protective isolation to the falling number of remission deaths in our series. Children who have been in contact with measles and chicken pox may be partially protected by pooled or specific gammaglobulin.

Treatment of bacterial, fungal, and pneumocystis infection is possible with antibiotics and other chemotherapeutic agents, and when these fail to control the infection in neutropenic patients, white cell transfusions are now of proven benefit (Graw and Appelbaum, 1977). It is possible that we averted one remission death in 1976 by white cell transfusions.

Viral infections may be emerging as an important contribution to the mortality of children with ALL. Rapid ante-mortem diagnosis of viral infections will enable clinicians to take full advantage of effective antiviral agents as they become available.

However, within the framework of existing antileukaemic therapy schedules, the most important factor in prevention of death from infection is the acquisition of clinical expertise in treating children with ALL. This can only be achieved by the centralization of treatment and by the co-operation and sharing of knowledge between treatment centres.

We are grateful to Dr. W. Walker and Dr. T. C. Noble for allowing us to report patients under their care, and for encouragement and guidance. Dr. D. J. Scott performed most of the necropsy examinations and we are grateful for permission to study these. M.M.R. and E.B. are supported by grants from the Tyneside Leukaemia Research Association.

\section{References}

Abdallah, P. S., Mark, J. B., and Merigan, T. C. (1976). Diagnosis of cytomegalovirus pneumonia in compromised hosts. American Journal of Medicine, 61, 326-332.

British Medical Journal (1976). Measles encephalitis during immunosuppressive treatment, 1, 1552.

Craft, A. W., Reid, M. M., and Low, W. T. (1976). The effect of virus infections on polymorph function in children. British Medical Journal, 1, 1570.

Esber, E., DiNicola, W., Movassaghi, N., and Leikin, S. (1976). $T$ and $B$ lymphocytes in leukemia therapy. American Journal of Hematology, 1, 211-218.

Feigin, R. D., and Shearer, W. T. (1975). Opportunistic infection in children. II. In the compromised host. Journal of Pediatrics, 87, 677-694.

Gardner, P. S., and McQuillin, J. (1974). Rapid Virus Diagnosis: Application of Immunofluorescence. Butterworths, London.

Gardner, P. S., Turk, D. C., Aherne, W. A., Bird, T., Holdaway, M. D., and Court, S. D. M. (1967). Deaths associated with respiratory tract infection in childhood. British Medical Journal, 4, 316-320.

Graham-Pole, J. G., Davie, M., Kershaw, I., Barter, D. A. C., and Willoughby, M. L. N. (1976). Granulocyte transfusion in treatment of infected neutropenic children. Archives of Disease in Childhood, 51, 521-527.

Graw, R. G., and Appelbaum, S. R. (1977). Granulocyte transfusion therapy-a review of the practical aspects of collection and transfusion techniques. Experimental Hematology, 5, Suppl., 39-48. 
Holland, J. F., and Glidewell, O. (1972). Oncologists' reply: survival expectancy in acute lymphocytic leukemia. New England Journal of Medicine, 287, 769-777.

Hughes, W. T. (1971). Fatal infections in childhood leukemia. American Journal of Diseases of Children, 122, 283-287.

Hughes, W. T. (1976). Treatment of pneumocystis carinii pneumonitis. New England Journal of Medicine, 295, 726-727.

Hughes, W. T., and Smith, D. R. (1973). Infection during induction of remission in acute lymphocytic leukemia. Cancer, 31, 1008-1014.

Johnston, P. G. B., Hardisty, R. M., Kay, H. E. M., and Smith, P. G. (1974). Myelosuppressive effect of colaspase (L-asparaginase) in initial treatment of acute lymphoblastic leukaemia. British Medical Journal, 3, 81-83.

MacLennan, I. C. M., Kay, H. E. M., Festenstein, M., and Smith, P. G. (1976). Analysis of treatment in childhood leukaemia. II. Timing and toxicity of combined 6-mercaptopurine and methotrexate maintenance therapy. British Journal of Haematology, 33, 179-188.

Medical Research Council Working Party on Leukaemia in Childhood (1975). Analysis of treatment in childhood leukaemia. I. British Medical Journal, 3, 563-566.

Murphy, J. V., and Yunis, E. J. (1976). Encephalopathy following measles infection in children with chronic illness. Journal of Pediatrics, 88, 937-942.

Pearson, T. A., Mitchell, C. A., and Hughes, W. T. (1972) Aeromonas hydrophila septicemia. American Journal of Diseases of Children, 123, 579-582.

Pullan, C. R., Noble, T. C., Scott, D. J., Wisniewski, K., and Gardner, P. S. (1976). Atypical measles infections in leukaemic children on immunosuppressive treatment. British Medical Journal, 1, 1562-1565.

Reid, M. M., Craft, A. W., and Todd, J. A. (1977). Serial studies of numbers of circulating $T$ and $B$ lymphocytes in children with acute lymphoblastic leukaemia. Archives of Disease in Childhood, 52, 245-247.

Simone, J. V., Aur, R. J. A., Hustu, H. O., Verzosa, M., and Pinkel, D. (1975). Combined modality therapy of acute lymphocytic leukemia. Cancer, 35, 25-35.

Sims, D. G., Scott, D. J., and Noble, T. C. (1976). Multiple major cerebral artery thromboses with profound thrombocytopenia in acute leukaemia. Archives of Disease in Childhood, 51, 74-77.

Correspondence to Dr. A. W. Craft, Department of Child Health, Royal Victoria Infirmary, Newcastle upon Tyne NE1 4LP. 